\title{
Staged versus conventional nursing for patients receiving chemotherapy for advanced non-small cell lung cancer: a before and after study
}

\author{
Leijuan Zhu ${ }^{1 \#}$, Lijia Chen ${ }^{1 \#}$, Haifeng Kan ${ }^{1}$, Pengfei Cai ${ }^{2}$ \\ ${ }^{1}$ Respiratory Department, Rugao People's Hospital, Rugao, China; ${ }^{2}$ Imaging Department, Rugao People's Hospital, Rugao, China \\ Contributions: (I) Conception and design: L Zhu, L Chen, H Kan, P Cai; (II) Administrative support: P Cai; (III) Provision of study materials or \\ patients: L Zhu, L Chen; (IV) Collection and assembly of data: L Zhu, L Chen; (V) Data analysis and interpretation: L Zhu, L Chen, H Kan; (VI) \\ Manuscript writing: All authors; (VII) Final approval of manuscript: All authors. \\ \#These authors contributed equally to this work. \\ Correspondence to: Haifeng Kan. Respiratory Department, Rugao People's Hospital, No. 278, Ninghai Road, Rugao 226500, China. \\ Email: 43203323@163.com; Pengfei Cai. Imaging Department, Rugao People’s Hospital, No. 278, Ninghai Road, Rugao 226500 , China. \\ Email: clj656778@sina.com.
}

Background: Advanced non-small cell lung cancer (NSCLC) patients receiving chemotherapy usually
experience adverse events, especially chemotherapy-induced nausea and vomiting (CINV), which reduces
their quality of life (QoL). The present before and after study was performed to investigate the influence of
graded nursing on adverse events and QoL of advanced NSCLC patients. Methods: Patients with stage III or IV NSCLC who received conventional nursing from January 2017 to December 2017 were selected as the control group. Patients with stage III or IV NSCLC who received staged nursing from January 2018 to December 2018 were selected as the study group. Adverse events, especially CINV, were recorded as a primary outcome of this study. The QoL of patients was assessed using the European Organization for Research and Treatment of Cancer (EORTC) quality of life questionnaire (QLQ)-C30 scale. All patients were followed up for at least 1 year.

Results: Patients showed no significant difference in baseline clinical characteristics between the control and study groups. Adverse events, including vomiting, nausea, constipation, fever, cough, mucositis, and epigastric pain, occurred, of which CINV was the most common. However, no difference in these adverse events was found between the 2 groups. The EORTC QLQ-C30 scale, which assesses the physical, role, emotional, cognitive, and social functions and global health status, indicated no significant difference between the 2 groups prior to receiving chemotherapy. Expectedly, patients in the study group had improved emotional, cognitive, and social function and global health status compared with the control group $(\mathrm{P}=0.004$, $\mathrm{P}=0.017, \mathrm{P}=0.008$, and $\mathrm{P}=0.003$, respectively). Additionally, the Kaplan-Meier survival curve indicated that patients in the study group had a statistically better survival rate than patients in the control group $(\mathrm{P}=0.032)$. Graded nursing was found to be a protective factor of overall survival of advanced NSCLC patients according to the Cox proportional hazards model $(\mathrm{P}=0.024)$.

Conclusions: Graded nursing could significantly improve QoL and prolong the survival of advanced NSCLC patients receiving chemotherapy, and may be a feasible measurement to improve their prognosis.

Koywords: Staged nursing; non-small cell lung cancer (NSCLC); chemotherapy; quality of life (QoL); adverse events; overall survival

Submitted Oct 12, 2020. Accepted for publication Dec 02, 2020.

doi: 10.21037/apm-20-2240

View this article at: http://dx.doi.org/10.21037/apm-20-2240 


\section{Introduction}

Lung cancer is the most commonly diagnosed cancer worldwide, according to the World Health Organization (1), with more than 1.4 million new cases and 1.3 million deaths each year (2). Non-small cell lung cancer (NSCLC) is the most common of lung cancer, accounting for around $80 \%$ of all lung cancers (3). Despite some improvements in the early diagnosis of lung cancer in recent decades, most NSCLC patients are still diagnosed late (stage IIIB or IV) due to NSCLC being usually asymptomatic and non-specific in the early stage (4). These patients have a particularly poor prognosis, with an estimated 5-year survival rate of no more than $4.5 \%$ (5). The main therapeutic goal of NSCLC is to reduce symptoms, increase survival, and improve quality of life (QoL).

Palliative chemotherapy remains the first-line treatment for late-stage NSCLC patients, and may extend the survival rate by several weeks or months. However, palliative chemotherapy also has many side-effects (6). Of these, chemotherapy-induced nausea and vomiting (CINV) is one of the most common and distressing side-effects among NSCLC patients (7). Chemotherapy-induced nausea refers to the desire to vomit with or without tachycardia, dizziness, and weakness, and vomiting refers to the reflux of gastric contents induced by chemotherapeutic agents (8). It has been reported that approximately $35 \%$ and $60 \%$ of patients experience acute or delayed CINV, respectively (9). Persistent CINV significantly affects the confidence and QoL of patients.

A balance between effectiveness and tolerance of NSCLC treatment is important. Intolerance of treatment and occurrence of adverse events, especially CINV, will significantly affect the QoL of cancer patients and further reduce their willingness to continue chemotherapy (10). Some efforts have been made to reduce the side-effects of chemotherapy and improve the QoL of NSCLC patients, such as combination chemotherapy, the development of targeted agents, and the use of immune-checkpoint inhibitors (11), which reduce some side-effects to a certain extent $(12,13)$. Only a few studies have investigated the role of nursing in the management of NSCLC patients receiving chemotherapy. In their study, Boucher et al. confirmed that structured nursing intervention with NSCLC patients is important to enhance their proper oral chemotherapeutic adherence (14). Recently, the concept of individualized graded nursing was proposed to improve the recovery of patients. Ma et al. reported the positive effects of individualized graded nursing on blood coagulation function in children with nephritis (15). However, no related study was performed to explore the relationship between staged nursing and QoL of NSCLC patients.

Therefore, we conducted this before and after study to determine the effects of staged nursing on the incidence of CINV and QoL in NSCLC patients receiving chemotherapy.

We present the following article in accordance with the STROBE reporting checklist (available at http://dx.doi. org/10.21037/apm-20-2240).

\section{Methods}

\section{Patient selection}

The present study was an observational before and after study conducted in Rugao People's Hospital (Rugao, China). Patients with stage III or IV NSCLC who received conventional nursing care from January 2017 to December 2017 were selected as the control group. Patients with stage III or IV NSCLC who received staged nursing care from January 2018 to December 2018 were selected as the study group. The inclusion criteria were as follows: (I) adult patients with stage III or IV NSCLC; and (II) patients receiving conventional chemotherapy. The exclusion criteria were as follows: (I) patients who underwent surgical procedure; (II) patients who were administrated drugs which could affect the study results. The data of patients in the control group were retrospectively collected; therefore, signed informed consent was not required. Patients in the study group provided signed informed consent prior to data collection. The study was performed in compliance with the ethical principles of the Declaration of Helsinki (as revised in 2013), and was approved by the Ethics Committee of Rugao People's Hospital (No. 20190032).

\section{Data collection}

Data of patients in both groups were collected, including their age, sex, stage of NSCLC, pathological type, chemotherapy scheme, and Karnofsky Performance Status (KPS). The KPS score was used in the present study to measure the physical status of NSCLC patients, and ranged from 0 to 100 points.

\section{Nursing management}

Patients in the control group received routine nursing 
measures, including basic nursing, psychological nursing, and health education, to ensure the safety of medication during chemotherapy and to prevent the occurrence of adverse effects.

In the study group, patients were divided into 3 grades according to their KPS score: grade I: 80-100, representing self-care; grade II: $60-80$, representing part self-care; and grade III: 0-60, representing the patient's inability to take care of himself. Based on their grade, patients received different nursing managements in addition to conventional nursing care. The focus of nursing management for grade I patients was health education to promote recovery after chemotherapy and avoid serious chemotherapy-related adverse events. Changes in each patient's condition were monitored. For grade II patients, the focus was to encourage physical activities and to enhance their psychological care; assistance was provided. For grade III patients, the focus was to monitor changes in patients' conditions and their vital signs; patients were kept as comfortable as possible. The occurrence of adverse events was detected quickly, and symptomatic treatment was provided.

\section{Outcomes}

Adverse events, especially CINV, were recorded as a primary outcome in the present study. Additionally, the European Organization for Research and Treatment of Cancer (EORTC) quality of life questionnaire (QLQ)-C30 scale (version 3.0, Chinese version) was used to assess the overall QoL of patients and the impact of disease and treatment. All patients were followed up for at least 1 year, and their condition was also recorded.

\section{Statistical analysis}

SPSS (version 20.0; SPSS, Armonk, NY, USA) was used for the statistical analysis. Continuous data were reported as means \pm standard deviations. Categorical data were expressed as numbers with percentages. Two-tailed $t$-test and $\chi^{2}$-test were used to compare the differences between continuous data and categorical data, respectively. The Kaplan-Meier survival curve of NSCLC patients was generated using GraphPad Prism (version 7.0; GraphPad Software, San Diego, CA, USA), and the log-rank test was used to compare the difference between the control and study groups. The Cox proportional hazards model was used to assess the risk factors for overall survival in advanced NSCLC patients. $\mathrm{P}<0.05$ indicated statistical significance.

\section{Results}

Based on the inclusion and exclusion criteria, 71 advanced NSCLC patients were retrospectively enrolled in the study as the control group of conventional nursing care. Fifty-five patients receiving chemotherapy between January 2018 and December 2018 were prospectively included as the study group of staged nursing care. The clinical characteristics of patients in both groups are shown in Table 1. The majority of NSCLC patients were male; there were $43(60.6 \%)$ male patients in the control group and $36(65.5 \%)$ in the study group, respectively. Approximately half the patients were $45-65$ years old, and $22(31 \%)$ were $>65$ years in the control group. In the study group, 32 (58.2\%) patients were aged 45-65 years. Most patients in both groups were diagnosed with stage IV NSCLC. With regard to pathological type, approximately half the NSCLC cases were adenocarcinoma, with $38(53.5 \%)$ in the control group and $27(49.1 \%)$ in the study group. All patients received platinumbased chemotherapy. The KPS scores of patients before chemotherapy were also recorded, with a mean score of $76.4 \pm 12.5$ in the control group and $74.9 \pm 10.8$ in the study group.

Adverse events, including vomiting, nausea, constipation, fever, cough, mucositis, and epigastric pain, are shown in Table 2. CINV was found to be the most common adverse event. Seventeen $(23.9 \%)$ patients experienced vomiting in the control study, whereas $11(20.0 \%)$ experienced vomiting in the study group. Fourteen $(19.7 \%)$ patients experienced vomiting in the control study, whereas 8 $(14.5 \%)$ experienced vomiting in the study group. However, no differences were found in adverse events between the 2 groups (Table 2).

QoL was assessed using the EORTC QLQ-C30 scale, including physical, role, emotional, cognitive, and social function and global health status. There were no significant differences between the 2 groups in various functions and global health status before receiving chemotherapy (Table 3). Expectedly, patients in the study group who received staged nursing care had improved emotional, cognitive, and social function and global health status ( $\mathrm{P}=0.004, \mathrm{P}=0.017, \mathrm{P}=0.008$, and $\mathrm{P}=0.003$, respectively).

All patients were followed up for at least 1 year, and overall survival was recorded (Figure 1). The results of the Gehan-Wilcoxon test suggested that patients in the study group had a statistically better survival rate compared with the control group $(\mathrm{P}=0.032)$. The Cox proportional hazards model was also used to assess risk factors for overall 
Table 1 Clinical characteristics of patients in the control and study groups

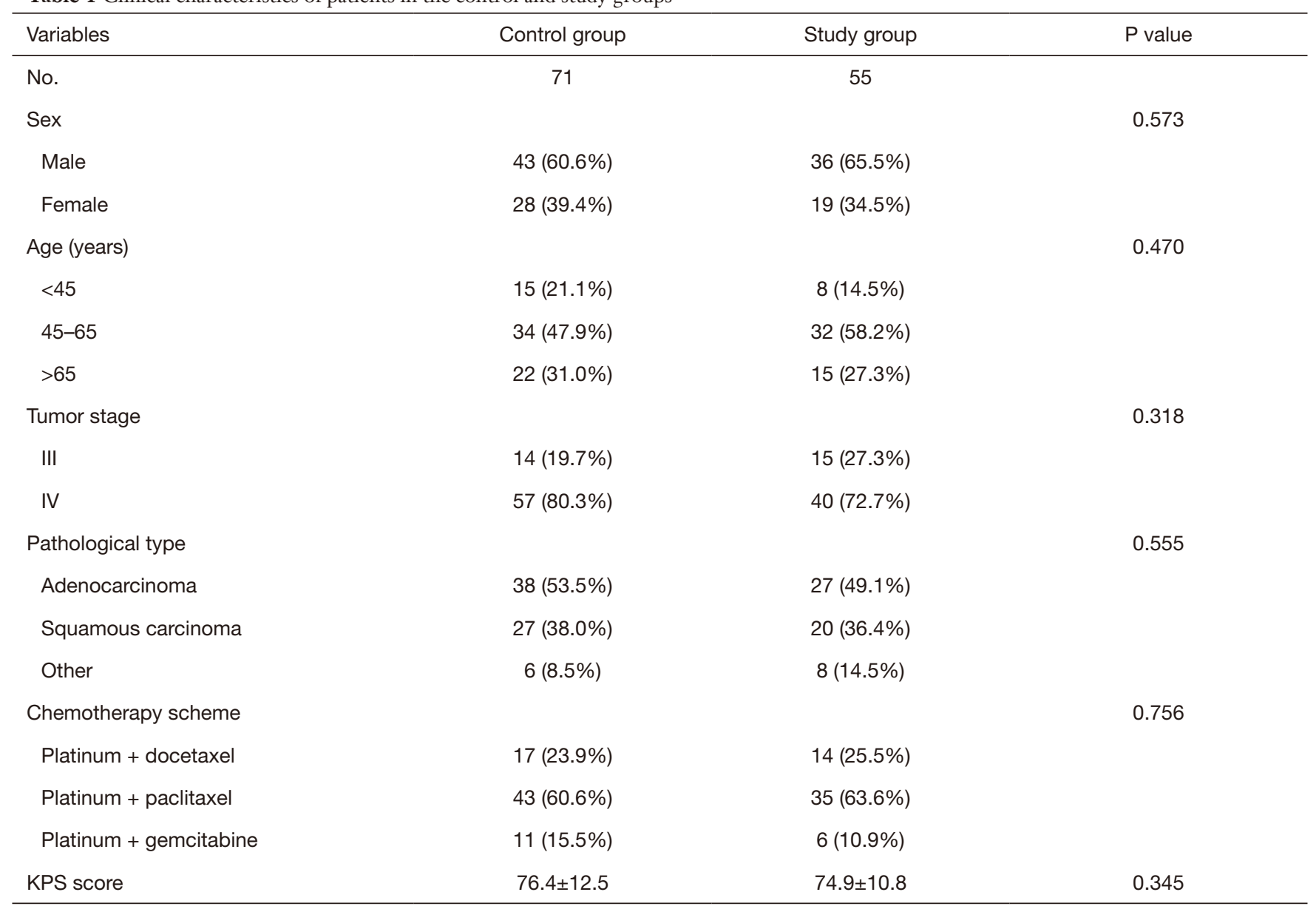

KPS, Karnofsky Performance Status.

Table 2 Adverse events in the control and study groups

\begin{tabular}{lccc}
\hline Variables & Control group & Study group & $c$ value \\
\hline Vomiting & $17(23.9 \%)$ & $11(20.0 \%)$ & 0.597 \\
Nausea & $14(19.7 \%)$ & $8(14.5 \%)$ & 0.448 \\
Constipation & $10(14.1 \%)$ & $7(12.7 \%)$ & 0.825 \\
Fever & $10(14.1 \%)$ & $5(9.1 \%)$ & 0.391 \\
Cough & $5(7.0 \%)$ & $4(7.3 \%)$ & 0.960 \\
Mucositis & $2(2.8 \%)$ & $2(3.6 \%)$ & 0.795 \\
Epigastric pain & $2(2.8 \%)$ & $4(7.3 \%)$ & 0.244 \\
\hline
\end{tabular}

survival (Table 4). After adjusting by multivariate analysis, graded nursing was found to be a protective factor of overall survival of advanced NSCLC patients $(\mathrm{P}=0.024$, hazards ratio: $0.558,95 \%$ confidence interval: $0.336-0.926)$. In addition, age, tumor stage, and the EORTC QLQ-C30 scale global health status were also confirmed to be associated with the overall survival of advanced NSCLC patients. 
Table 3 Changes in quality of life before and after chemotherapy in the control and study groups according to the European Organization for Research and Treatment of Cancer quality of life questionnaire (QLQ)-C30 scale

\begin{tabular}{|c|c|c|c|c|c|c|}
\hline Variables & \multicolumn{2}{|c|}{ Control group } & \multicolumn{2}{|c|}{ Study group } & $P$ value ${ }^{a}$ & $P$ value ${ }^{b}$ \\
\hline Physical function & $75.6 \pm 18.4$ & $76.9 \pm 19.2$ & $73.9 \pm 20.5$ & $76.8 \pm 22.3$ & 0.197 & 0.480 \\
\hline Role function & $81.5 \pm 22.5$ & $82.6 \pm 21.4$ & $81.4 \pm 19.7$ & $83.1 \pm 14.7$ & 0.649 & 0.206 \\
\hline Emotional function & $65.0 \pm 25.8$ & $67.9 \pm 20.5$ & $63.7 \pm 24.1$ & $77.3 \pm 19.6$ & 0.340 & 0.004 \\
\hline Social function & $76.5 \pm 20.2$ & $77.9 \pm 21.6$ & $77.6 \pm 16.2$ & $83.9 \pm 19.8$ & 0.476 & 0.008 \\
\hline Global health status & $58.4 \pm 19.5$ & $59.3 \pm 21.0$ & $55.3 \pm 22.6$ & $65.4 \pm 20.7$ & 0.712 & 0.003 \\
\hline
\end{tabular}

${ }^{a}$, control group before chemotherapy vs. study group before chemotherapy; ${ }^{b}$, control group after chemotherapy vs. study group after chemotherapy.

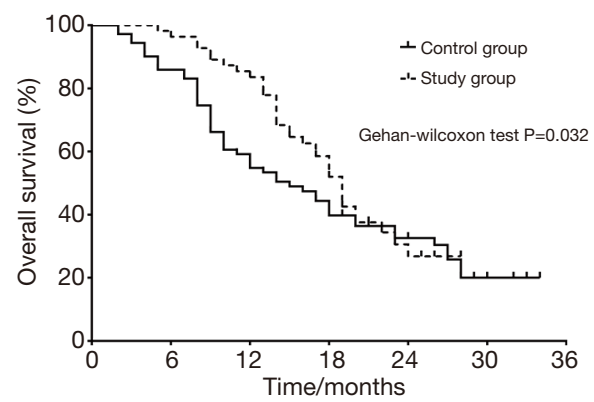

Figure 1 Kaplan-Meier survival curve of non-small cell lung cancer patients in the control and study groups.

\section{Discussion}

The majority of NSCLC patients receiving chemotherapy experience various adverse events, especially CINV, which will influence their QoL. However, there are few studies focusing on measurements to reduce adverse events and improving QoL. Staged nursing care was a novel measurement to provide individualized nursing care to cancer patients. The aim of the present study was to explore the impact of graded nursing on adverse events and QoL of NSCLC patients. It was found that graded nursing significantly improved QoL, although no association was found between graded nursing and adverse events. Graded nursing was also found to prolong the survival of advanced NSCLC patients receiving chemotherapy.

The most common adverse event in the present study was CINV, in which vomiting was the most significant $[\mathrm{n}=17(23.9 \%)$ in the control group; $\mathrm{n}=11(20 \%)$ in the study group]. It has been previously reported that sex has a significant impact on adverse events induced by chemotherapy (16). Wheatley-Price et al. found that female patients experienced more CINV compared with male patients ( $77 \%$ vs. $66 \%, \mathrm{P}=0.0004)(17)$. Age also plays a vital role in the incidence of adverse events, with older NSCLC patients being more likely to experience adverse events (18). We initially assumed, prior to undertaking this study, that grading nursing may be able to reduce adverse events of advanced NSCLC patients receiving chemotherapy. However, the results indicated that the incidence of adverse events during chemotherapy was more likely to depend on the individual's response to the drugs and the rate of drug metabolism.

Although graded nursing did not exhibit any significant impact on the incidence of adverse events, it still significantly improved the QoL of NSCLC patients in our study. There are a number of previously published studies exploring efficient measurements to improve the QoL of NSCLC patients (19-27). The co-administration of conventional chemotherapeutic agents and novel drugs provides an option. Several previously published studies have reported that this can improve the QoL of NSCLC patients $(23,25,27)$. Adamowicz et al. also proposed that the level of patient knowledge of cancer was associated with QoL (21). Another study also indicated that behavioral activation, a measurement that encourages patients to maintain rewarding activities to activate their sources of day-to-day reinforcement, could be a feasible intervention to improve their social and role function (28). The results of our study are in accordance with these studies. The main advantage of graded nursing is that it can provide patients closer condition monitoring, impart more NSCLC-related 
Table 4 Univariate and multivariate analyses of risk factors for overall survival among non-small cell lung cancer patients

\begin{tabular}{|c|c|c|c|}
\hline Risk factors & $\begin{array}{c}\text { Univariate analysis } \\
\mathrm{P} \text { value }\end{array}$ & \multicolumn{2}{|c|}{ Multivariate analysis } \\
\hline Graded nursing versus conventional nursing & 0.029 & $0.558(0.336-0.926)$ & 0.024 \\
\hline Sex: male vs. female & 0.292 & - & - \\
\hline Age (years): $>65$ vs. $<65$ & 0.001 & $3.170(1.239-8.108)$ & 0.016 \\
\hline Pathological type: adenocarcinoma vs. other & 0.139 & - & - \\
\hline Chemotherapy scheme: platinum + paclitaxel vs. other & 0.233 & - & - \\
\hline Karnofsky Performance Status score & 0.006 & $0.993(0.979-1.007)$ & 0.314 \\
\hline Global health status of EORTC QLQ-C30 scale & 0.001 & $0.972(0.950-0.994)$ & 0.014 \\
\hline
\end{tabular}

$\mathrm{Cl}$, confidence interval; EORTC, European Organization for Research and Treatment of Cancer; HR, hazard ratio.

knowledge, and help patients with appropriate physical exercise. Psychological care is also essential for such patients, and grade nursing encourages patients to return to the hospital for treatment and to better tolerate drug sideeffects.

A greater willingness for treatment and better drug tolerance further improves the treatment effect of patients. The Kaplan-Meier survival curve indicated relatively better overall survival in the study group compared with control group, especially in the first 18 months. However, the overall effect of treatment for advanced NSCLC is not satisfactory due to the rapid progression and metastasis of lung cancer and its low sensitivity to chemotherapeutic agents. The survival rate of patients in both groups was similar after 18 months. Therefore, lung cancer still needs better treatment measurements and novel drugs in the long term, although graded care can improve the prognosis of patients in the short term.

The main limitation of the present study was small sample size, which may limit the findings, and future largescale clinical studies are warranted. Second, the data of the control group were collected retrospectively; therefore, there may be some errors in the data, resulting in bias in the final results. The strength of the study was the homogeneity of the patients in terms of sex, age, tumor stage, pathological type, chemotherapy scheme, and KPS score.

\section{Conclusions}

The aim of the present before and after study was to investigate the impact of graded nursing on adverse events and QoL of advanced NSCLC patients receiving chemotherapy. The results indicated that graded nursing provides no significant benefit to the reduction of adverse events. However, graded nursing was found to significantly improve QoL and prolong the survival of advanced NSCLC patients. The present study provides some evidence for the application of graded nursing in clinical practice, and graded nursing may be a feasible measurement to improve the prognosis of advanced NSCLC patients receiving chemotherapy.

\section{Acknowledgments}

Funding: None.

\section{Footnote}

Reporting Checklist: The authors have completed the STROBE reporting checklist. Available at http://dx.doi. org/10.21037/apm-20-2240

Data Sharing Statement: Available at http://dx.doi. org/10.21037/apm-20-2240

Conflicts of Interest: All authors have completed the ICMJE uniform disclosure form (available at http://dx.doi. org/10.21037/apm-20-2240). The authors have no conflicts of interest to declare.

Ethical Statement: The authors are accountable for all 
aspects of the work in ensuring that questions related to the accuracy or integrity of any part of the work are appropriately investigated and resolved. The data of patients in the control group were retrospectively collected; therefore, signed informed consent was not required. Patients in the study group provided signed informed consent prior to data collection. The study was performed in compliance with the ethical principles of the Declaration of Helsinki (as revised in 2013), and was approved by the Ethics Committee of Rugao People's Hospital (No. 20190032).

Open Access Statement: This is an Open Access article distributed in accordance with the Creative Commons Attribution-NonCommercial-NoDerivs 4.0 International License (CC BY-NC-ND 4.0), which permits the noncommercial replication and distribution of the article with the strict proviso that no changes or edits are made and the original work is properly cited (including links to both the formal publication through the relevant DOI and the license). See: https://creativecommons.org/licenses/by-nc-nd/4.0/.

\section{References}

1. Bray F, Ferlay J, Soerjomataram I, et al. Global cancer statistics 2018: GLOBOCAN estimates of incidence and mortality worldwide for 36 cancers in 185 countries. CA Cancer J Clin 2018;68:394-424.

2. De Angelis R, Sant M, Coleman MP, et al. Cancer survival in Europe 1999-2007 by country and age: results of EUROCARE--5-a population-based study. Lancet Oncol 2014;15:23-34.

3. Couillard-Montminy V, Gagnon PY, Fortin S, et al. Effectiveness of adjuvant carboplatin-based chemotherapy compared to cisplatin in non-small cell lung cancer. J Oncol Pharm Pract 2019;25:44-51.

4. Arenberg D. Update on screening for lung cancer. Transl Lung Cancer Res 2019;8:S77-87.

5. Russell J, Wong ML, Mackin L, et al. Stability of Symptom Clusters in Patients With Lung Cancer Receiving Chemotherapy. J Pain Symptom Manage 2019;57:909-22.

6. Wang Z, Cai XJ, Chen LY, et al. Factors potentially associated with gemcitabine-based chemotherapy-induced thrombocytopenia in Chinese patients with nonsmall cell lung cancer. J Cancer Res Ther 2018;14:S656-60.

7. Sommariva S, Pongiglione B, Tarricone R. Impact of chemotherapy-induced nausea and vomiting on healthrelated quality of life and resource utilization: A systematic review. Crit Rev Oncol Hematol 2016;99:13-36.

8. Carnio S, Galetta D, Scotti V, et al. Chemotherapyinduced nausea and vomiting (CINV) in patients with advanced lung cancer during the first-line treatment: assessment by physicians, nurses, and patients from an Italian multicenter survey. Support Care Cancer 2018;26:1841-9.

9. Ihbe-Heffinger A, Ehlken B, Bernard R, et al. The impact of delayed chemotherapy-induced nausea and vomiting on patients, health resource utilization and costs in German cancer centers. Ann Oncol 2004;15:526-36.

10. Ying M, Liu J, Zhou W, et al. The Role of Additional Chemotherapy in Combination with Concurrent Chemoradiotherapy for Locally Advanced Inoperable Non-Small Cell Lung Cancer, a Systematic Review and Meta-Analysis of 12 Randomized Trials. Cancer Invest 2019;37:376-86.

11. Tun AM, Thein KZ, Thein WL, et al. Checkpoint inhibitors plus chemotherapy for first-line treatment of advanced non-small cell lung cancer: a systematic review and meta-analysis of randomized controlled trials. Future Sci OA 2019;5:FSO421.

12. Sun JM, Lee KH, Kim BS, et al. Pazopanib maintenance after first-line etoposide and platinum chemotherapy in patients with extensive disease small-cell lung cancer: a multicentre, randomised, placebo-controlled Phase II study (KCSG-LU12-07). Br J Cancer 2018;118:648-53.

13. Ishii H, Azuma K, Kawahara A, et al. Atezolizumab plus carboplatin and etoposide in small cell lung cancer patients previously treated with platinum-based chemotherapy. Invest New Drugs 2020. [Epub ahead of print]. doi: 10.1007/s10637-020-00983-6.

14. Boucher J, Lucca J, Hooper C, et al. A Structured Nursing Intervention to Address Oral Chemotherapy Adherence in Patients With Non-Small Cell Lung Cancer. Oncol Nurs Forum 2015;42:383-9.

15. Ma H, Yuan M, Zhu H. Positive effect of individualized grading nursing on blood coagulation function in children with henoch schonlein purpura nephritis. Minerva Med 2020. [Epub ahead of print]. doi: 10.23736/S00264806.20.06906-2.

16. Tsiouda T, Sardeli C, Porpodis K, et al. Sex Differences and Adverse Effects between Chemotherapy and Immunotherapy for Non-Small Cell Lung Cancer. J Cancer 2020;11:3407-15.

17. Wheatley-Price P, Le Maitre A, Ding K, et al. The influence of sex on efficacy, adverse events, quality of life, and delivery of treatment in National Cancer Institute of 
Canada Clinical Trials Group non-small cell lung cancer chemotherapy trials. J Thorac Oncol 2010;5:640-8.

18. Chrischilles EA, Pendergast JF, Kahn KL, et al. Adverse events among the elderly receiving chemotherapy for advanced non-small-cell lung cancer. J Clin Oncol 2010;28:620-7.

19. Yao J, Jiao L, Yao Y, et al. The effect of comprehensive rehabilitation program plus chemotherapy on quality of life in patients with postoperative non-small-cell lung cancer: study protocol of a multicenter randomized clinical trial. Trials 2020;21:309.

20. Belloumi N, Maalej Bellaj S, Bachouche I, et al. Comparison of Sleep Quality before and after Chemotherapy in Locally Advanced Nonsmall Cell Lung Cancer Patients: A Prospective Study. Sleep Disord 2020;2020:8235238.

21. Adamowicz K, Janiszewska J, Lichodziejewska-Niemierko M. Prognostic Value of Patient Knowledge of Cancer on Quality of Life in Advanced Lung Cancer During Chemotherapy. J Cancer Educ 2020;35:93-9.

22. Papadopoulos D, Kiagia M, Charpidou A, et al. Psychological correlates of sleep quality in lung cancer patients under chemotherapy: A single-center crosssectional study. Psychooncology 2019;28:1879-86.

23. Morabito A, Piccirillo MC, Maione P, et al. Effect on quality of life of cisplatin added to single-agent chemotherapy as first-line treatment for elderly patients

Cite this article as: Zhu L, Chen L, Kan H, Cai P. Staged versus conventional nursing for patients receiving chemotherapy for advanced non-small cell lung cancer: a before and after study. Ann Palliat Med 2021;10(1):250-257. doi: 10.21037/apm20-2240 with advanced non-small cell lung cancer: Joint analysis of MILES-3 and MILES-4 randomised phase 3 trials. Lung Cancer 2019;133:62-8.

24. Check JH, Check D, Poretta T. Mifepristone Extends Both Length and Quality of Life in a Patient With Advanced Non-small Cell Lung Cancer that Has Progressed Despite Chemotherapy and a Check-point Inhibitor. Anticancer Res 2019;39:1923-6.

25. Tremmas I, Petsatodis G, Potoupnis M, et al. Monitoring changes in quality of life in patients with lung cancer under treatment with chemotherapy and co administration of zoledronic acid by using specialized questionnaires. J Cancer 2018;9:1731-6.

26. Udupa K, Rajendranath R, Sagar TG. Comparison study of quality of life in advanced lung cancer patients on tyrosine kinase inhibitor and platinum doublet chemotherapy. Indian J Cancer 2017;54:161-3.

27. Liu J, Mao JJ, Li SQ, et al. Preliminary Efficacy and Safety of Reishi \& Privet Formula on Quality of Life Among Non-Small Cell Lung Cancer Patients Undergoing Chemotherapy: A Randomized Placebo-Controlled Trial. Integr Cancer Ther 2020;19:1534735420944491.

28. Fernandez-Rodriguez C, Villoria-Fernandez E, Fernandez-Garcia P, et al. Effects of Behavioral Activation on the Quality of Life and Emotional State of Lung Cancer and Breast Cancer Patients During Chemotherapy Treatment. Behav Modif 2019;43:151-80. 